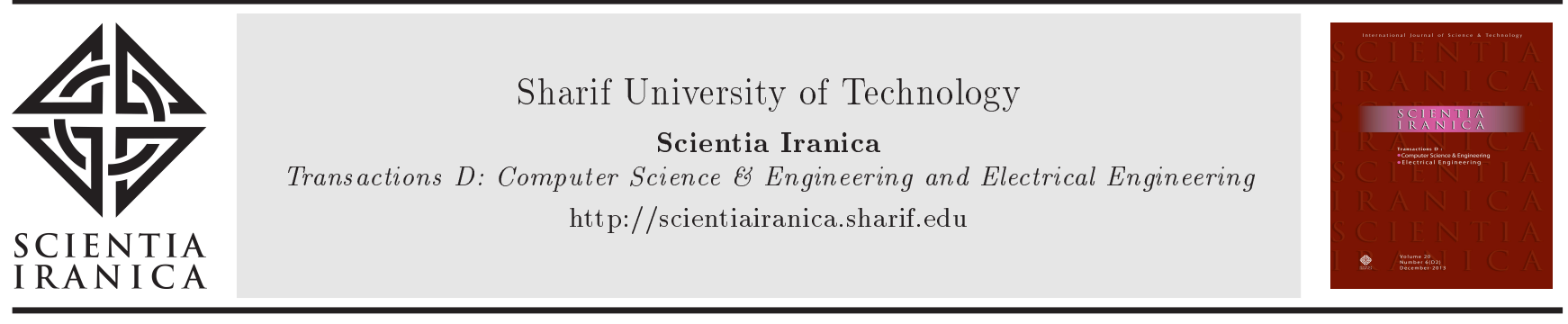

\title{
Analytic solution of a system of linear distributed order differential equations in the Reimann-Liouville sense
}

\author{
H. Taghavian and M.S. Tavazoei* \\ Department of Electrical Engineering Sharif University of Technology, Tehran, Iran.
}

Received 17 August 2016; received in revised form 17 December 2017; accepted 23 April 2018

\author{
KEYWORDS \\ Analytic solution; \\ Distributed order \\ differential equation; \\ Reimann-Liouville \\ fractional derivative; \\ Mittag-Leffler \\ function; \\ Relaxation process.
}

\begin{abstract}
In this paper, the solution to a system of linear distributed order differential equations in the Riemann-Liouville sense is analytically obtained. The distributed order relaxation equation is a special case of the system investigated in this paper. The solution of the mentioned system is introduced on the basis of a function, which can be considered as the distributed order generalization of the matrix Mittag-Leffler functions. It is shown that this generalized function in two special cases of the weight function can be expressed in terms of the multivariate Mittag-Leffler functions and the Wright functions.
\end{abstract}

(C) 2020 Sharif University of Technology. All rights reserved.

\section{Introduction}

Fractional order calculus is used to model physical processes that exhibit anomalous dynamics, such as fractional order diffusion [1,2] and relaxation [3]. Relaxation processes modeled by integer order or fractional order derivatives could be generalized by the concept of distributed order derivatives that allows modeling processes with scaling law change [4]. In addition, this could be done by utilizing either Riemann-Liouville or Caputo definition to construct a distributed order differentiation operator for modeling distributed order relaxation. Although the two approaches turn out to be equivalent in the fractional order case, this is not true in the distributed order case. Since both approaches lead to successful models describing the actual physical phenomena, a further study of both cases is appealing. The typical approach to obtaining a solution to such equations in the time domain is based on direct inverse

\footnotetext{
*. Corresponding author. Tel.: +98 2166165930 ;

Fax: +982166023261

E-mail address: tavazoei@sharif.edu (M.S. Tavazoei)
}

Laplace transform using the Fourier-Mellin formula or Titchmarsh theorem [5]. This approach, which is considered in most papers [4,6-9] in the literature, generates a solution expressed by a Laplace-type integral in both Riemann-Liouville and Caputo cases. Since the link between this representation and the FoxWright functions used in fractional order differential equations is not clear, an alternative representation of the solution, which incorporates Fox-Wright functions, is proposed in [9] in which the Laplace-type integral still lingers. This problem was also studied by using Laguerre series to give an approximation of the solution in [10]. In addition, the asymptotic properties of the solution is investigated in [6]. This problem is also treated in the case of triple impulses and double impulses as special cases of the weight function in [4] and [11], respectively. It is observed that a coherent extension of the Mittag-Leffler functions for distributed order calculus is of interest. In this regard, a new representation of the solution associated with the general weight functions is presented, which excludes the Laplace-type integral. This representation consists of a series expansion, which exhibits the impact of the weight function on the distribution of the orders in the time domain. The reduction of the solution to the 
Mittag-Leffler function is clear in the case of a single impulse weight function. Moreover, it is shown that the solution turns into the multivariate Mittag-Leffler function in the case of several impulses as the weight function. On the other hand, by choosing a unitary or exponential weight function, it is possible to express the solution in terms of the Wright functions.

This paper is organized as follows. In Section 2, some preliminaries are reviewed, and some useful lemmas are presented with regard to the distributed order calculus. Section 3 is devoted to the main results, where the solution of a system of linear distributed order differential equations in the Riemann-Liouville sense is presented in terms of what can be construed as the distributed order generalization of Mittag-Leffler functions. Some properties of these functions are stated, and the problem is investigated further in two special cases of the weight functions. At the end, numerical examples are presented to simulate the solution to a distributed order relaxation equation and a system of differential equations with multiple fractional order operators as special cases in Section 4. Finally, the paper is concluded in Section 5.

\section{Distributed order calculus}

In this section, some definitions and lemmas are presented that are essential to achieving the main results in the rest of the paper. First off, some of the notations used in this paper are introduced as follows:

\section{Notation}

$\begin{array}{ll}w & \text { Weight function } \\ W_{\alpha, \beta} & \text { Wright function } \\ E_{\alpha, \beta} & \text { Mittag-Leffler function } \\ E_{\left(\beta_{1}, \beta_{2}, \ldots, \beta_{n}\right), \sigma} & \text { Multivariate Mittag-Leffler function } \\ { }_{p} \Psi_{q} & \text { Fox-Wright function } \\ \delta & \text { Dirac delta function } \\ H & \text { Heaviside step function } \\ \Gamma & \text { Complete Gamma function } \\ { }_{0} I_{t}^{\alpha} & \text { Fractional integral } \\ { }_{0}^{C} D_{t}^{\alpha} & \text { Fractional derivative in the Caputo } \\ { }_{0} L & \text { Sense } \\ D_{t}^{\alpha} & \text { Fractional derivative in the Riemann- } \\ { }_{0} I_{t}^{w(\alpha)} & \text { Liouville sense } \\ R L & \text { Distributed order integral } \\ { }_{0} D_{t}^{w(\alpha)} & \text { Distributed order derivative in the } \\ * & \text { Riemann-Liouville sense } \\ f^{* k} & \text { Convolution } \\ L_{t \rightarrow s} & k \text {-fold iteration of convolution }\end{array}$

The cornerstone of fractional calculus is based on the extension of integration order to real numbers in integral operators. This is realized by introducing a fractional integration operator [12, P. 65].

$$
{ }_{0} I_{t}^{\alpha} f(t)=\int_{0}^{t}\left((t-\tau)^{\alpha-1} / \Gamma(\alpha)\right) f(\tau) d \tau .
$$

The Caputo fractional derivative is defined based on this definition as follows [12]:

$$
{ }_{0}^{C} D_{t}^{\alpha} f(t)= \begin{cases}f(t), & \alpha=0 \\ { }_{0} I_{t}^{1-\alpha} \dot{f}(t), & 0<\alpha<1 . \\ \dot{f}(t), & \alpha=1\end{cases}
$$

In this paper, the Riemann-Liouville fractional derivative is considered, which is defined as follows [12]:

$$
{ }_{0}^{R L} D_{t}^{\alpha} f(t)= \begin{cases}f(t), & \alpha=0 \\ \frac{d}{d t} 0 I_{t}^{1-\alpha} f(t), & 0<\alpha<1 . \\ \dot{f}(t), & \alpha=1\end{cases}
$$

The Riemann-Liouville fractional derivative (3) is equivalent to the Caputo fractional derivative (2) if the function subject to the operator has zero initial conditions [12]. Integrating operator (1) over the order of integration with a weight function results in the socalled distributed order integral [13]:

$$
{ }_{0} I_{t}^{w(\alpha)} f(t)=\int_{0}^{1} w(\alpha)_{0} I_{t}^{\alpha} f(t) d \alpha .
$$

In the definition above, $w: \mathbb{R} \rightarrow \mathbb{R}$ denotes the weight function, for which $w(\alpha)=0$ holds for $\alpha \in$ $(-\infty, 0] \cup[1,+\infty)$. Distributed order derivative can also be defined in a similar way:

$$
{ }_{0}^{R L} D_{t}^{w(\alpha)} f(t)=\int_{0}^{1} w(\alpha)_{0}^{R L} D_{t}^{\alpha} f(t) d \alpha .
$$

Power functions with real powers tend to appear in fractional calculus frequently. In addition, the Laplace transform of these functions is given as follows [14]:

$$
L_{t \rightarrow s}\left\{t^{a}\right\}=\Gamma(a+1) / s^{a+1}, \quad a \in(-1,+\infty) .
$$

The Riemann-Liouville fractional derivative of the power function is given by [12]:

$$
\begin{gathered}
{ }_{0}^{R L} D_{t}^{\alpha} t^{\mu}=\Gamma(\mu+1) t^{\mu-\alpha} / \Gamma(\mu-\alpha+1), \\
t>0, \quad \mu>-1 .
\end{gathered}
$$

According to Eq. (6), the representation of fractional integral of a function in the Laplace domain is given by [12]:

$$
L_{t \rightarrow s}\left\{{ }_{0} I_{t}^{\alpha} f(t)\right\}=F(s) / s^{\alpha},
$$


where $F(s)=L_{t \rightarrow s}\{f(t)\}$. In addition, the representation of the fractional derivative of a function with zero initial conditions in the Laplace domain is given by [12]:

$$
L_{t \rightarrow s}\left\{\left\{_{0}^{R L} D_{t}^{\alpha} f(t)\right\}=s^{\alpha} F(s) .\right.
$$

Moreover, the following Laplace transformation is required:

$$
L_{t \rightarrow s}\{-\ln t-\gamma\}=(\ln s) / s,
$$

where $\gamma$ is the Euler-Mascheroni constant [14]. Before proceeding, some of the special functions used in fractional calculus are reviewed. It has been shown that the solution to linear differential equations of fractional order with constant coefficients is expressed in terms of Mittag-Leffler functions [12]. The Mittag-Leffler function is defined by the series [12].

$$
E_{\alpha, \beta}(z)=\sum_{k=0}^{+\infty} z^{\alpha k} / \Gamma(\alpha k+\beta), \quad \alpha, \beta>0, \quad z \in \mathbb{C} .
$$

In fact, Function (11) has been generalized in several ways to fit different problems in fractional calculus. A generalization of Eq. (11), which appears in the solution to fractional differential equations by the operational methods, is the multivariate Mittag-Leffler Function [15]. This function is defined by:

$$
\begin{aligned}
E_{\left(\beta_{1}, \beta_{2}, \ldots, \beta_{n}\right), \sigma}\left(z_{1}, z_{2}, \ldots, z_{n}\right) & =\sum_{k=0}^{+\infty} \\
\sum_{l_{1}+l_{2}+\cdots+l_{n}=k}\left(\begin{array}{c}
k \\
l_{1}, l_{2}, \cdots, l_{n}
\end{array}\right) & \frac{\prod_{i=1}^{n} z_{i}^{l_{i}}}{\Gamma\left(\sum_{i=1}^{n} l_{i} \beta_{i}+\sigma\right)},
\end{aligned}
$$

where $\left(\begin{array}{c}k \\ l_{1}, l_{2}, \cdots, l_{n}\end{array}\right)=\frac{k !}{l_{1} ! l_{2} ! \cdots l_{n} !}$. The Wright function, which frequently appears in fractional order diffusion-wave equations, is defined by the series [12]:

$$
\begin{aligned}
& W_{\alpha, \beta}(z)=\sum_{k=0}^{+\infty} \frac{z^{k}}{k ! \Gamma(\alpha k+\beta)}, \\
& \alpha, \beta>0 \quad z \in \mathbb{C} .
\end{aligned}
$$

Wright functions and Mittag-Leffler functions are both generalized by the following definition:

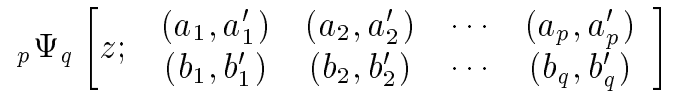

$$
\begin{aligned}
& =\sum_{k=0}^{+\infty} \frac{\Gamma\left(a_{1}+a_{1}^{\prime} k\right) \Gamma\left(a_{2}+a_{2}^{\prime} k\right) \cdots \Gamma\left(a_{p}+a_{p}^{\prime} k\right) z^{k}}{\Gamma\left(b_{1}+b_{1}^{\prime} k\right) \Gamma\left(b_{2}+b_{2}^{\prime} k\right) \cdots \Gamma\left(b_{q}+b_{q}^{\prime} k\right) k !},
\end{aligned}
$$

which is called the Fox-Wright function [16].

\section{Proposition 1}

The representation of the distributed order integral of a function in the Laplace domain is given by:

$$
L_{t \rightarrow s}\left\{{ }_{0} I_{t}^{w(\alpha)} f(t)\right\}=\left.L_{\alpha \rightarrow \hat{s}}\{w(\alpha)\}\right|_{\hat{s}=\ln s} F(s),
$$

where $F(s)=L_{t \rightarrow s}\{f(t)\}$.

Proof

Considering the Laplace transform of Eq. (4) and using Eq. (8) result in:

$$
L_{t \rightarrow s}\left\{{ }_{0} I_{t}^{\alpha} f(t)\right\}=\int_{0}^{1} w(\alpha) F(s) / s^{\alpha} d \alpha .
$$

Using the relation $1 / s^{\alpha}=\exp (-\alpha \operatorname{In} s)$ in Eq. (16), we obtain:

$$
\begin{aligned}
L_{t \rightarrow s} & \left\{{ }_{0} I_{t}^{w(\alpha)} f(t)\right\} \\
& =\left(\int_{0}^{+\infty} w(\alpha) \exp (-\alpha \ln s) d \alpha\right) F(s) \\
& =\left.L_{\alpha \rightarrow \hat{s}}\{w(\alpha)\}\right|_{\hat{s}=\ln s} F(s) .
\end{aligned}
$$

\section{Proposition 2}

The representation of the distributed order derivative of a function with zero initial conditions in the Laplace domain is given by:

$$
L_{t \rightarrow s}\left\{\begin{array}{l}
R L \\
0
\end{array} D_{t}^{w(\alpha)} f(t)\right\}=\left.L_{\alpha \rightarrow \hat{s}}\{w(\alpha)\}\right|_{\hat{s}=-\ln s} F(s),
$$

where $F(s)=L_{t \rightarrow s}\{f(t)\}$.

Proof of Proposition 2 is obtained as easily as that of Proposition 1. We continue this section with an extension of the fractional power function $t^{a}, a \in$ $(-1,+\infty)$ by introducing a distributed power function, which turns out to be a remarkably general function, resulting in various well-known functions as its special cases. This function is defined by:

$$
p(t ; f) \triangleq \int_{0}^{+\infty}\left(t^{\alpha-1} / \Gamma(\alpha)\right) f(\alpha) d \alpha,
$$

where $f: \mathbb{R} \rightarrow \mathbb{R}$ is a locally integrable function satisfying $f(\alpha)=0$ for $\alpha \in(-\infty, 0]$. It is observed that $p(t ; \Gamma(\alpha) \delta(\alpha-a-1))=t^{a}$, indicating how the fractional power function is a special case of this function. It is shown that this is also true for the more general FoxWright functions in the following proposition.

\section{Proposition 3}

It can be shown that:

1. $p\left(t ; \sum_{k=0}^{+\infty} \delta\left(\alpha-k \beta_{1}-\beta_{2}\right)\right)=$

$$
t^{\beta_{2}-1} E_{\beta_{1}, \beta_{2}}\left(t^{\beta_{1}}\right), \quad \beta_{1}, \beta_{2}>0,
$$


2. $p\left(t ; \sum_{k=0}^{+\infty} \delta\left(\alpha-k \beta_{1}-\beta_{2}\right) / k !\right)$

$$
=t^{\beta_{2}-1} W_{\beta_{1}, \beta_{2}}\left(t^{\beta_{1}}\right), \quad \beta_{1}, \beta_{2}>0,
$$

3. $\quad p\left(t ; \sum_{k=0}^{+\infty} \delta(\alpha-k-1)\right.$

$$
\begin{aligned}
& \left.\frac{\Gamma\left(a_{1}+a_{1}{ }_{1} k\right) \Gamma\left(a_{2}+a_{2}{ }_{2} k\right) \cdots \Gamma\left(a_{p}+a_{p}^{\prime} k\right)}{\Gamma\left(b_{1}+b^{\prime}{ }_{1} k\right) \Gamma\left(b_{2}+b^{\prime}{ }_{2} k\right) \cdots \Gamma\left(b_{q}+b^{\prime}{ }_{q} k\right)}\right) \\
& ={ }_{p} \Psi_{q}\left[t ; \begin{array}{rrrr}
\left(a_{1}, a_{1}^{\prime}\right) & \left(a_{2}, a_{2}^{\prime}\right) & \cdots & \left(a_{p}, a_{p}^{\prime}\right) \\
\left(b_{1}, b_{1}^{\prime}\right) & \left(b_{2}, b_{2}^{\prime}\right) & \cdots & \left(b_{q}, b_{q}^{\prime}\right)
\end{array}\right] .
\end{aligned}
$$

\section{Proof}

Proof is directly obtained from definition (Eq. (19)).

The Laplace transform of distributed order power functions is given in the following lemma.

\section{Lemma 1}

Let $f: \mathbb{R} \rightarrow \mathbb{R}$ be a locally integrable function satisfying $f(\alpha)=0$ for $\alpha \in(-\infty, 0]$. Then, $L_{t \rightarrow s}\{p(t ; f(\alpha))\}=F(\ln s)$, where $F(s)=L_{t \rightarrow s}\{f(t)\}$.

\section{Proof}

Using Eq. (6), Laplace transform of Eq. (19) is given by:

$$
L_{t \rightarrow s}\{p(t ; f(\alpha))\}=\int_{0}^{+\infty}\left(f(\alpha) / s^{\alpha}\right) d \alpha .
$$

Using the relation $1 / s^{\alpha}=\exp (-\alpha \ln s)$ in Eq. (20), we obtain:

$$
\begin{aligned}
L_{t \rightarrow s}\{p(t ; f(\alpha))\} & =\int_{0}^{+\infty} f(\alpha) \exp (-\alpha \ln s) d \alpha \\
& =F(\ln s),
\end{aligned}
$$

where $F(s)=L_{\alpha \rightarrow s}\{f(\alpha)\}$.

Distributed order integrals of distributed power functions can be calculated by the following lemma.

\section{Lemma 2}

Let $f: \mathbb{R} \rightarrow \mathbb{R}$ be a locally integrable function satisfying $f(\alpha)=0$ for $\alpha \in(-\infty, 0]$. Then:

$$
{ }_{0} I_{t}^{w(\alpha)} p(t ; f(\alpha))=p(t ; w(\alpha) * f(\alpha)),
$$

where $w(\alpha) * f(\alpha)=\int_{0}^{\alpha} w(\alpha-\tau) f(\tau) d \tau$.

\section{Proof}

According to Proposition 1,

$$
\begin{aligned}
L_{t \rightarrow s} & \left\{0 I_{t}^{w(\alpha)} p(t ; f(\alpha))\right\} \\
& =\left.L_{\alpha \rightarrow \hat{s}}\{w(\alpha)\}\right|_{\hat{s}=\ln s} L_{t \rightarrow s}\{p(t ; f(\alpha))\} .
\end{aligned}
$$

By using Lemma 1, Eq. (23) yields

$$
\begin{gathered}
L_{t \rightarrow s}\left\{{ }_{0} I_{t}^{w(\alpha)} p(t ; f(\alpha))\right\}=\left.L_{\alpha \rightarrow \hat{s}}\{w(\alpha)\}\right|_{\hat{s}=\ln s} F(\ln s) \\
=L_{t \rightarrow s}\{p(t ; w * f(\alpha))\},
\end{gathered}
$$

which results in Eq. (22).

The following lemma is used to determine the distributed order derivatives of distributed power functions.

\section{Lemma 3}

Let $f: \mathbb{R} \rightarrow \mathbb{R}$ be a locally integrable function $f(\alpha)=0$ for $\alpha \in(-\infty, 1]$. Then:

$$
{ }_{0}^{R L} D_{t}^{w(\alpha)} p(t ; f(\alpha))=p(t ; w(-\alpha) * f(\alpha)),
$$

where $w(-\alpha) * f(\alpha)=\int_{0}^{1+\alpha} w(\tau-\alpha) f(\tau) d \tau$.

\section{Proof}

Considering the Laplace transform of the left side of Eq. (25) and using Proposition 2 give:

$$
\begin{aligned}
L_{t \rightarrow s} & \left\{{ }_{0}^{R L} D_{t}^{w(\alpha)} p(t ; f(\alpha))\right\} \\
& =\left.L_{\alpha \rightarrow \hat{s}}\{w(\alpha)\}\right|_{\hat{s}=-\ln s} L_{t \rightarrow s}\{p(t ; f(\alpha))\} .
\end{aligned}
$$

Using Lemma 1 yields:

$$
\begin{aligned}
L_{t \rightarrow s}\left\{\begin{array}{l}
R L \\
0
\end{array} D_{t}^{w(\alpha)} p(t ; f(\alpha))\right\} \\
\quad=\left.L_{\alpha \rightarrow \hat{s}}\{w(\alpha)\}\right|_{\hat{s}=-\ln s} F(\ln s) .
\end{aligned}
$$

With respect to the relation $L_{\alpha \rightarrow s}\{w(-\alpha) * f(\alpha)\}=$ $\left.L_{\alpha \rightarrow \hat{s}}\{w(\alpha)\}\right|_{\hat{s}=-s} F(s)$ and using Lemma 1, it is concluded that:

$$
\begin{aligned}
& \left.L_{\alpha \rightarrow \hat{s}}\{w(\alpha)\}\right|_{\hat{s}=-\ln s} F(\ln s) \\
& \quad=L_{t \rightarrow s}\{p(t ; w(-\alpha) * f(\alpha))\},
\end{aligned}
$$

which is the Laplace transform of the right side of Eq. (25).

\section{Distributed order system of linear differential equations}

The distributed order system of linear differential equations is supposed to be a generalization of a fractional order system of linear differential equations. This generalization may be done in two ways such that extending the fractional order system to distributed order one is possible by using Caputo or Riemann-Liouville operators. In fact, the actual physical processes of interest, such as diffusion phenomena and relaxation patterns, can be modeled by either of the operators successfully [6,17]. The distributed order system of linear differential equations in the Riemann-Liouville sense is introduced as follows: 


$$
\begin{aligned}
& \dot{x}(t)=A \int_{0}^{1} w(\alpha)_{0}^{R L} D_{t}^{1-\alpha} x(t) d \alpha+B g(t), \\
& x(0)=x_{0}
\end{aligned}
$$

where $x(t) \in \mathbb{R}^{n}$ is the pseudo state, and $g: \mathbb{R} \rightarrow \mathbb{R}$ is a locally integrable function satisfying $g(t)=0$ for $t \in(-\infty, 0)$. In addition, $A \in \mathbb{R}^{n \times n}$ and $B \in \mathbb{R}^{n \times 1}$. Likewise, the distributed order system described in the Caputo sense is defined by:

$$
\begin{aligned}
& \int_{0}^{1} w(\alpha)_{0}^{C} D_{t}^{\alpha} x(t) d \alpha=A x(t)+B u(t), \\
& x(0)=x_{0},
\end{aligned}
$$

which was comprehensively studied in [18]. This study is concerned with the Riemann-Liouville-based system (Eq. (29)), and it aims to present the analytical solution for such a system. It is obvious that in the case of scalar negative $A$ and $g(t) \equiv 0$, this system is reduced to a distributed order relaxation equation [6]. We recall that Riemann-Liouville derivative (Eq. (3)) is the left inverse of the fractional integral operator (Eq. (1)). It is interesting that this relationship does not hold for distributed order operators (Eqs. (4) and (5)) in general. This is the fundamental reason why modeling distributed order relaxation patterns by means of Caputo and Riemann-Liouville operators does not produce identical solutions.

This section is followed by definition below, which specifies the iterated self-convolution of a function:

$$
f^{* k}(t)=\int_{0}^{t} f(\tau) f^{* k-1}(t-\tau) d \tau, \quad k \in \mathbb{N} .
$$

In Eq. (31), $f: \mathbb{R} \rightarrow \mathbb{R}$ is a locally integrable function satisfying $f(t)=0$ for $t \in(-\infty, 0]$; for the initial function, we define $f^{* 0}(t)=\delta(t)$. Eq. (31) is sometimes called the convolution power of function $f(t)$, which has some applications in stochastic differential equations [19]. Based on this definition, a function that can be considered as the distributed order version of the Mittag-Leffler function is introduced. This function, like the Fox Wright function, is also a special case of the distributed power function (Eq. (19)) and is defined by:

$$
E(t ; A w(\alpha)) \triangleq p(t ; v(\alpha))
$$

where:

$$
v(\alpha+1)=\sum_{k=0}^{+\infty} A^{k} w^{* k}(\alpha) .
$$

According to this definition and noting the fact that $w^{* k}(\alpha)$ is zero outside the range $(0, k)$, this function can be represented by:

$$
\begin{aligned}
E(t ; A w(\alpha))= & \sum_{k=0}^{+\infty} \int_{0}^{k} A^{k} w^{* k}(\alpha) \\
& \left(t^{\alpha} / \Gamma(\alpha+1)\right) d \alpha .
\end{aligned}
$$

\section{Lemma 4}

The exact solution to the homogeneous system of differential equations:

$$
\dot{x}(t)=A \int_{0}^{1} w(\alpha)_{0}^{R L} D_{t}^{1-\alpha} x(t) d \alpha, \quad x(0)=x_{0},
$$

is given by:

$$
x(t)=E(t ; A w(\alpha)) x_{0}, \quad t \geq 0,
$$

where $E(t ; A w(\alpha))$ is defined by Eq. (32).

\section{Proof}

Defining:

$$
v_{1}(\alpha+1)=\sum_{k=1}^{+\infty} A^{k} w^{* k}(\alpha),
$$

allows us to write $v(\alpha+1)=\delta(\alpha)+v_{1}(\alpha+1)$. Thereby:

$$
E(t ; A w(\alpha))=p\left(t ; \delta(\alpha-1)+v_{1}(\alpha)\right) .
$$

Accordingly, following Eq. (19), the distributed power function is linear with respect to its second argument. In addition, it is deduced from Eq. (19) that $p(t ; \delta(\alpha-1))=1$. Therefore:

$$
E(t ; A w(\alpha))=1+p\left(t ; v_{1}(\alpha)\right) .
$$

To show that $x(t)=E(t ; A w(\alpha)) x_{0}$ is the solution of Eq. (35), we need to prove:

$$
\begin{aligned}
& \frac{d}{d t} E(t ; A w(\alpha)) x_{0} \\
& \quad=A \int_{0}^{1} w(\alpha)_{0}^{R L} D_{t}^{1-\alpha} E(t ; A w(\alpha)) x_{0} d \alpha .
\end{aligned}
$$

The right side of Eq. (40) equals:

$$
\begin{array}{rl}
A \int_{0}^{1} & w(\alpha)_{0}^{R L} D_{t}^{1-\alpha} E(t ; A w(\alpha)) d \alpha x_{0} \\
= & A \int_{0}^{1} w(\alpha)_{0}^{R L} D_{t}^{1-\alpha}(1) d \alpha x_{0} \\
& +A \int_{0}^{1} w(\alpha)_{0}^{R L} D_{t}^{1-\alpha} p\left(t ; v_{1}(\alpha)\right) d \alpha x_{0} .
\end{array}
$$

Using Eq. (7) for the calculation of the first term in the second line of Eq. (41), we obtain: 


$$
\begin{gathered}
A \int_{0}^{1} w(\alpha)_{0}^{R L} D_{t}^{1-\alpha}(1) d \alpha x_{0}=A \int_{0}^{1} w(\alpha) \\
t^{\alpha-1} / \Gamma(\alpha) d \alpha x_{0}=p(t ; A w(\alpha)) x_{0} .
\end{gathered}
$$

For the second term in the second line of Eq. (41), the variable change, $\beta=1-\alpha$, is considered, which gives:

$$
\begin{gathered}
A \int_{0}^{1} w(1-\beta)_{0}^{R L} D_{t}^{\beta} p\left(t ; v_{1}(\alpha)\right) d \beta x_{0} \\
=A_{0}^{R L} D_{t}^{w(1-\alpha)} p\left(t ; v_{1}(\alpha)\right) x_{0} .
\end{gathered}
$$

Note that $v_{1}(\alpha)=0$ for $0 \leq \alpha \leq 1$. Therefore, Lemma 3 is used to calculate $A_{0}^{R} L D_{t}^{w(1-\alpha)} p\left(t ; v_{1}(\alpha)\right) x_{0}$ as in the following:

$$
\begin{aligned}
& A_{0}^{R L} D_{t}^{w(1-\alpha)} p\left(t ; v_{1}(\alpha)\right) x_{0} \\
& \quad=A p\left(t ; w(1+\alpha) * v_{1}(\alpha)\right) x_{0} .
\end{aligned}
$$

Applying the time shift to the other function involved in the convolution, we obtain:

$$
\begin{aligned}
A_{0}^{R L} D_{t}^{w(1-\alpha)} p\left(t ; v_{1}(\alpha)\right) x_{0} \\
\quad=A p\left(t ; w(\alpha) * v_{1}(\alpha+1)\right) x_{0} .
\end{aligned}
$$

Thereby, using Eq. (33) gives:

$$
\begin{aligned}
A_{0}^{R L} & D_{t}^{w(1-\alpha)} p\left(t ; v_{1}(\alpha)\right) x_{0} \\
& =A p\left(t ; w(\alpha) * \sum_{k=1}^{+\infty} A^{k} w^{* k}(\alpha)\right) x_{0} \\
& =A p\left(t ; \sum_{k=1}^{+\infty} A^{k} w^{* k+1}(\alpha)\right) x_{0} \\
& =p\left(t ; \sum_{k=1}^{+\infty} A^{k+1} w^{* k+1}(\alpha)\right) x_{0} \\
& =p\left(t ; \sum_{k=2}^{+\infty} A^{k} w^{* k}(\alpha)\right) x_{0} .
\end{aligned}
$$

By using Eqs. (42) and (46), the right side of Eq. (40) can be written as follows:

$$
\begin{aligned}
& A \int_{0}^{1} w(\alpha)_{0}^{R L} D_{t}^{1-\alpha} E(t ; A w(\alpha)) d \alpha x_{0}=p(t ; A w(\alpha)) x_{0} \\
& \quad+p\left(t ; \sum_{k=2}^{+\infty} A^{k} w^{* k}(\alpha)\right) x_{0} \\
& \quad=p\left(t ; \sum_{k=1}^{+\infty} A^{k} w^{* k}(\alpha)\right) x_{0}
\end{aligned}
$$

On the other hand, for the left side of Eq. (40), one has:

$$
\frac{d}{d t} E(t ; A w(\alpha)) x_{0}=\frac{d}{d t} p\left(t ; v_{1}(\alpha)\right) x_{0}
$$

Due to the equality:

$$
\frac{d}{d t} p\left(t ; v_{1}(\alpha)\right)={ }_{0}^{R L} D_{t}^{\delta(\alpha-1)} p\left(t ; v_{1}(\alpha)\right),
$$

Lemma 3 is used to write the left side of Eq. (40) as follows:

$$
\frac{d}{d t} E(t ; A w(\alpha)) x_{0}=p\left(t ; \delta(-\alpha-1) * v_{1}(\alpha)\right) x_{0} .
$$

Since $\delta(\alpha)$ is an even function, we can write:

$$
\begin{aligned}
\frac{d}{d t} E(t ; A w(\alpha)) x_{0} & =p\left(t ; \delta(\alpha+1) * v_{1}(\alpha)\right) x_{0} \\
& =p\left(t ; v_{1}(\alpha+1)\right) x_{0} \\
& =p\left(t ; \sum_{k=1}^{+\infty} A^{k} w^{* k}(\alpha)\right) x_{0},
\end{aligned}
$$

which is equal to the right side of Eq. (47).

\section{Lemma 5}

The exact solution to the system of differential equations characterized by input function $g(t)$ and zero initial conditions,

$$
\begin{aligned}
& \dot{x}(t)=A \int_{0}^{1} w(\alpha)_{0}^{R L} D_{t}^{1-\alpha} x(t) d \alpha+B g(t), \\
& x(0)=0,
\end{aligned}
$$

is given by:

$$
x(t)=E(t ; A w(\alpha)) * B g(t), \quad t \geq 0,
$$

in which $E(t ; A w(\alpha))$ is defined by Eq. (32).

\section{Proof}

Proof of this lemma follows a procedure quite similar to that of Lemma 4. We simply show that Eq. (52) satisfies Eq. (51). According to Eq. (39), the proposed solution can be written as follows:

$$
x(t)=\left(1+p\left(t ; v_{1}(\alpha)\right)\right) * B g(t) .
$$

At first, the left side of Eq. (51) is calculated as follows:

$$
\begin{aligned}
\dot{x}(t) & =B g(t)+\frac{d}{d t} p\left(t ; v_{1}(\alpha)\right) * B g(t) \\
& =B g(t)+\frac{d}{d t} \int_{0}^{+\infty} p\left(t-\tau ; v_{1}(\alpha)\right) H(t-\tau) B g(\tau) d \tau \\
& =B g(t)+\int_{0}^{+\infty} \dot{p}\left(t-\tau ; v_{1}(\alpha)\right) H(t-\tau) B g(\tau) d \tau \\
& +\int_{0}^{+\infty} p\left(t-\tau ; v_{1}(\alpha)\right) \delta(t-\tau) B g(\tau) d \tau
\end{aligned}
$$




$$
\begin{aligned}
& =B g(t)+\int_{0}^{+\infty} \dot{p}\left(t-\tau ; v_{1}(\alpha)\right) H(t-\tau) \\
& B g(\tau) d \tau+p\left(0 ; v_{1}(\alpha)\right) B g(t) .
\end{aligned}
$$

Since $v_{1}(\alpha)=0$ for $0 \leq \alpha \leq 1$, it is observed from Eq. (19) that $p\left(0 ; v_{1}(\alpha)\right)=0$. Therefore:

$$
\dot{x}(t)=B g(t)+\dot{p}\left(t ; v_{1}(\alpha)\right) * B g(\tau) \text {. }
$$

The term $\dot{p}\left(t ; v_{1}(\alpha)\right)$ has already been calculated in Eq. (50). Hence, for the left side of Eq. (51), we have:

$$
\begin{aligned}
\dot{x}(t) & =B g(t)+p\left(t ; v_{1}(\alpha+1)\right) * B g(\tau) \\
& =B g(t)+p\left(t ; \sum_{k=1}^{+\infty} A^{k} w^{* k}(\alpha)\right) * B g(t) .
\end{aligned}
$$

To calculate the right side of Eq. (51) using Eq. (39), we could write:

$$
\begin{aligned}
A \int_{0}^{1} w(\alpha)_{0}^{R L} D_{t}^{1-\alpha} x(t) d \alpha+B g(t) \\
=A \int_{0}^{1} w(\alpha)_{0}^{R L} D_{t}^{1-\alpha} I_{0}^{1} B g(t) d \alpha \\
\quad+A \int_{0}^{1} w(\alpha)_{0}^{R L} D_{t}^{1-\alpha}\left(p\left(t ; v_{1}(\alpha)\right)\right. \\
\quad * B g(t)) d \alpha+B g(t) .
\end{aligned}
$$

Since the relation ${ }_{0}^{R L} D_{t}^{1-\alpha}{ }_{0} I_{t}^{1}={ }_{0} I_{t}^{\alpha}$ [12] holds for the Riemann-Liouville derivative and fractional integral, we can write the following by expanding convolution integrals:

$$
\begin{aligned}
& A \int_{0}^{1} w(\alpha)_{0}^{R L} D_{t}^{1-\alpha} x(t) d \alpha+B g(t) \\
& =A \int_{0}^{1} w(\alpha) \int_{0}^{t}\left((t-\tau)^{\alpha-1} / \Gamma(\alpha)\right) B g(\tau) d \tau d \alpha \\
& \quad+A \int_{0}^{1} w(\alpha)_{0}^{R L} D_{t}^{1-\alpha}\left(\int_{0}^{+\infty} p\left(t-\tau ; v_{1}(\alpha)\right)\right. \\
& \quad H(t-\tau) B g(\tau) d \tau) d \alpha+B g(t) .
\end{aligned}
$$

By changing the order of integrations on the assumption that they exist, we obtain:

$$
\begin{aligned}
A \int_{0}^{1} w(\alpha)_{0}^{R L} D_{t}^{1-\alpha} x(t) d \alpha+B g(t) \\
=A \int_{0}^{t} \int_{0}^{1} w(\alpha)\left((t-\tau)^{\alpha-1} / \Gamma(\alpha)\right) d \alpha B g(\tau) d \tau \\
\quad+A \int_{0}^{+\infty} \int_{0}^{1} w(\alpha)_{0}^{R L} D_{t}^{1-\alpha}\left(p\left(t-\tau ; v_{1}(\alpha)\right)\right. \\
\quad H(t-\tau)) d \alpha B g(\tau) d \tau+B g(t) .
\end{aligned}
$$

Eq. (59) can be written in a distributed order derivative form as follows:

$$
\begin{aligned}
A \int_{0}^{1} w(\alpha)_{0}^{R L} D_{t}^{1-\alpha} x(t) d \alpha+B g(t) \\
=A p(t ; w(\alpha)) * B g(t) \\
\quad+A\left({ }_{0}^{R L} D_{t}^{w(1-\alpha)} p\left(t ; v_{1}(\alpha)\right)\right) \\
\quad * B g(t)+B g(t) .
\end{aligned}
$$

Using Lemma 3 yields:

$$
\begin{aligned}
& A \int_{0}^{1} w(\alpha)_{0}^{R L} D_{t}^{1-\alpha} x(t) d \alpha+B g(t) \\
&= A p(t ; w(\alpha)) * B g(t) \\
& \quad+A p\left(t ; w(\alpha+1) * v_{1}(\alpha)\right) * B g(t)+B g(t) \\
&= A p(t ; w(\alpha)) * B g(t) \\
&+A p\left(t ; w(\alpha) * v_{1}(\alpha+1)\right) * B g(t)+B g(t) .
\end{aligned}
$$

Finally, replacing $v_{1}(\alpha+1)$ into Eq. (37), we obtain the right side of Eq. (51) as follows:

$$
\begin{aligned}
A \int_{0}^{1} w(\alpha)_{0}^{R L} D_{t}^{1-\alpha} x(t) d \alpha+B g(t) \\
=p(t ; A w(\alpha)) * B g(t) \\
\quad+p\left(t ; \sum_{k=1}^{+\infty} A^{k+1} w^{* k+1}(\alpha)\right) * B g(t)+B g(t) \\
=p\left(t ; \sum_{k=1}^{+\infty} A^{k} w^{* k}(\alpha)\right) * B g(t)+B g(t), \quad(62)
\end{aligned}
$$

which is equal to Eq. (56).

\section{Theorem 1}

The exact solution to the system of differential (Eq. (29)) is given by:

$$
\begin{aligned}
& x(t)=E(t ; A w(\alpha)) x_{0}+E(t ; A w(\alpha)) * B g(t), \\
& t \geq 0,
\end{aligned}
$$

where $E(t ; A w(\alpha))$ is defined by Eq. (32).

\section{Proof}

Since Eq. (29) is linear, its solution is given by the summation of the homogeneous and particular solutions presented by Lemmas 4 and 5, respectively.

In order to highlight how the exact solution of a linear system of the distributed order differential 
equations is affected by the definition used for its differential operators, let us take a look at the system of differential equations in the Caputo sense (Eq. (30)). Considering Eq. (30) as an integral equation of convolution type, the resolvent formalism suggests the following representation for its solution:

$$
x(t)=\phi_{1}(t) x_{0}+\int_{0}^{t} \phi_{2}(t-\tau) B u^{\prime}(\tau) d \tau,
$$

where:

$$
\begin{aligned}
& \phi_{1}(t)=\int_{0}^{t} \sum_{k=0}^{+\infty} A^{k} i^{* k}(\tau) d \tau, \\
& \phi_{2}(t)=\int_{0}^{t} \sum_{k=0}^{+\infty} A^{k} i^{* k+1}(\tau) d \tau, \\
& u^{\prime}(t)=\frac{d}{d t} u(t), \\
& i(t)=L_{s \rightarrow t}^{-1}\left\{1 / \int_{0}^{1} w(\alpha) s^{\alpha} d \alpha\right\} .
\end{aligned}
$$

In fact, a variant of the distributed order integration can be defined as a linear time-invariant operator with impulse response $i(t)$. It can be shown that $i(t)$ cannot be expressed by elementary functions in general except a few special cases. For instance, in the case $w(\alpha) \geq$ 0 and $w(\alpha) \not \equiv 0$, by using the Fourier-Mellin inverse formula together with the residue theorem, $i(t)$ can be computed through an improper integral as follows:

$$
\begin{aligned}
& i(t)=\frac{1}{\pi} \int_{0}^{+\infty} \\
& \frac{\exp (-r t) \int_{0}^{1} w(\alpha) r^{\alpha} \sin (\pi \alpha) d \alpha}{\left(\int_{0}^{1} w(\alpha) r^{\alpha} \cos (\pi \alpha) d \alpha\right)^{2}+\left(\int_{0}^{1} w(\alpha) r^{\alpha} \sin (\pi \alpha) d \alpha\right)^{2}} d r .
\end{aligned}
$$

This representation of $i(t)$ can be achieved by following a procedure similar to the one employed in deriving the analytic solution of the diffusion problem in [8]. On the other hand, using the initial value theorem reveals that $i(t)$ has a singularity at $t=0$. Based on this argument, a comparison of the exact solutions of Eqs. (29) and (30) implies that using the RL definition for describing the distributed order system leads to an exact solution, which may be computed relatively easier. This is due to the fact that the calculation of convolution powers of the weight function, which is usually just a polynomial in the range of $[0,1]$, is obviously simpler than successive convolution powers of $i(t)$. In fact, $w^{* k}(\alpha)$ can be given explicitly for any $k \in \mathbb{N}$ in some usual cases of the weight function, and it can be efficiently computed using quadrature formulas.

\section{Lemma 6}

The Laplace transform of Function (32) is given by:

$$
\begin{aligned}
& L_{t \rightarrow s}\{E(t ; A w(\alpha))\}= \\
& \quad\left(I-\left.A \cdot L_{\alpha \rightarrow \hat{s}}\{w(\alpha)\}\right|_{\hat{s}=\ln s}\right)^{-1} / s .
\end{aligned}
$$

\section{Proof}

For convenience, the result of Lemma 5 is used, which states that Function (52) satisfies Eq. (51). Considering the Laplace transform of Eq. (51) by using Proposition 2 yields the following:

$$
s X(s)=A .\left.L_{\alpha \rightarrow \hat{s}}\{w(1-\alpha)\}\right|_{\hat{s}=-\ln s} X(s)+B G(s),
$$

where $G(s)=L_{\alpha \rightarrow s}\{g(t)\}$. Since $L_{\alpha \rightarrow s}\{w(1-\alpha)\}=$ $\left.L_{\alpha \rightarrow \hat{s}}\{w(\alpha)\}\right|_{\hat{s}=-s} \exp (-s)$, Eq. (65) takes the following form:

$$
s X(s)=\left.A L_{\alpha \rightarrow \hat{s}}\{w(\alpha)\}\right|_{\hat{s}=\ln s} s X(s)+B G(s) .
$$

Solving Eq. (66) with respect to $X(s)$ yields:

$$
X(s)=\left(I-\left.A L_{\alpha \rightarrow \hat{s}}\{w(\alpha)\}\right|_{\hat{s}=\ln s}\right)^{-1} B G(s) / s,
$$

where $I$ is the identity matrix with the same dimensions as matrix $A$. Since Eq. (67) is the Laplace transform of Eq. (52), this lemma follows.

In the following lemma, some properties of Function (32) are presented (in this lemma, it is shown that some properties of Function (32) are analogous to those of the Mittag-Leffler functions).

\section{Lemma 7}

Function (32) satisfies the following relations:

1. $E(t ; A \delta(\alpha-\beta))=E_{\beta, 1}\left(A t^{\beta}\right), \quad 0<\beta<1$.

2. ${ }_{0} I_{t}^{w(\alpha)} E(t ; A w(\alpha))=A^{-1}(E(t ; A w(\alpha))-I)$.

3. $E(a t ; A w(\alpha))=E\left(t ; A a^{\alpha} w(\alpha)\right), \quad a>0$.

4. $E(t ; A w(\alpha))+E(t ;-A w(\alpha))=2 E\left(t ; A^{2} w^{* 2}(\alpha)\right)$.

5. $\quad{ }_{0} I_{t}^{\frac{d}{d \alpha} w(\alpha)} E(t ; A w(\alpha))$

$$
=-A^{-1}(\ln t+\gamma) * \dot{E}(t ; A w(\alpha))
$$

\section{Proof}

1. Note that the iterated self-convolutions of the weight function become $w^{* k}(\alpha)=\delta(\alpha-\beta k)$ for this case; this result is followed by Eq. (34);

2. This result is followed by multiplying Eq. (64) by $\left(I-A .\left.L_{\alpha \rightarrow \hat{s}}\{w(\alpha)\}\right|_{\hat{s}=\ln s}\right)$ and rewriting the resultant expression in the time domain;

3. By writing $a^{\alpha}=\exp (\alpha \ln \alpha)$ and using the relation, $L_{\alpha \rightarrow s}\left\{a^{\alpha} w(\alpha)\right\}=\left.L_{\alpha \rightarrow \hat{s}}\{w(\alpha)\}\right|_{\hat{s}=s-\ln a}$, we have: 


$$
\begin{aligned}
L_{\alpha \rightarrow s}\{ & \left.E\left(t ; A a^{\alpha} w(\alpha)\right)\right\} \\
& =\frac{1}{s}\left(I-A L_{\alpha \rightarrow \hat{s}}\{w(\alpha)\}_{\mid \hat{s}=\ln (s)-\ln (a)}\right)^{-1} \\
& =\frac{1 / a}{s / a}\left(I-A L_{\alpha \rightarrow \hat{s}}\{w(\alpha)\}_{\mid \hat{s}=\ln (s / a)}\right)^{-1},
\end{aligned}
$$

which is equal to $L_{\alpha \rightarrow s}\{E(a t ; A w(\alpha))\}$.

4. Proof of this property is on the basis of the proof of the similar property for the Mittag-Leffler functions in [12]. In addition, it should be noted that although we have assumed the second argument of Function (32) to be a real matrix in our study, it is possible to define this function with complex arguments, too. The proof is presented for this generalized case. Since $\sum_{r=0}^{m-1} \exp (i 2 \pi k r / m)$ equals $m$ if $k \equiv 0(\bmod m)$ and zero otherwise, by using representation (34), we obtain:

$$
\begin{aligned}
\sum_{r=0}^{m-1} E(t ; A \exp (i 2 \pi r / m) w(\alpha)) & \int_{0}^{+\infty}\left(t^{\alpha} / \Gamma(\alpha+1)\right) \sum_{k=0}^{+\infty} A^{k} w^{* k}(\alpha) \\
& \sum_{r=0}^{m-1} \exp (i 2 \pi r k / m) d \alpha \\
= & \int_{0}^{+\infty}\left(t^{\alpha} / \Gamma(\alpha+1)\right) \\
& \sum_{k=0}^{+\infty} m A^{m k} w^{* m k}(\alpha) d \alpha \\
= & m E\left(t ; A^{m} w^{* m}(\alpha)\right) .
\end{aligned}
$$

As a special case, choosing $m=2$ in Eq. (69) gives the result of this part.

5. Multiplying and dividing Eq. (64) by In $s$ gives:

$$
\begin{aligned}
L_{t \rightarrow s} & \{E(t ; A w(\alpha))\} \\
& =\ln s\left(\ln s I-\left.A \ln s L_{\alpha \rightarrow \hat{s}}\{w(\alpha)\}\right|_{\hat{s}=\ln s}\right)^{-1} / s .
\end{aligned}
$$

Using the equality:

$$
\ln s L_{\alpha \rightarrow \hat{s}}\{w(\alpha)\}_{\mid \hat{s}=\ln s}=\left.L_{\alpha \rightarrow \hat{s}}\left\{\frac{d}{d \alpha} w(\alpha)\right\}\right|_{\hat{s}=\ln s}(71)
$$

we can rewrite Eq. (70) as follows:

$$
\begin{aligned}
& L_{t \rightarrow s}\{E(t ; A w(\alpha))\} \\
&=\ln s\left(\ln s I-\left.A L_{\alpha \rightarrow \hat{s}}\left\{\frac{d}{d \alpha} w(\alpha)\right\}\right|_{\hat{s}=\ln s}\right)_{(72)}^{-1 / s} .
\end{aligned}
$$

Multiplying Eq. (72) by: $\left(\ln s I-A L_{\alpha \rightarrow \hat{s}}\left\{\frac{d}{d \alpha} w(\alpha)\right\}\right.$ $\mid \hat{s}=\ln s)$ gives:

$$
\begin{aligned}
& (\ln s)(s / s) L_{t \rightarrow s}\{E(t ; A w(\alpha))\}-A L_{\alpha \rightarrow \hat{s}} \\
& \left.\left\{\frac{d}{d \alpha} w(\alpha)\right\}\right|_{\hat{s}=\ln s} L_{t \rightarrow s}\{E(t ; A w(\alpha))\} \\
& =I(\ln s) / s .
\end{aligned}
$$

By using Eq. (10) and the fact that $E(0 ; A w(\alpha))=$ $I$, the interpretation of $\mathrm{Eq}$. (73) in the time domain becomes:

$$
\begin{gathered}
-(\ln t+\gamma) *(\dot{E}(t ; A w(\alpha))+\delta(\alpha) I)-A_{0} I_{t}^{\frac{d}{d \alpha} w(\alpha)} \\
E(t ; A w(\alpha))=-(\ln t+\gamma) I .
\end{gathered}
$$

Based on Eq. (74), it is followed that:

$$
\begin{aligned}
-(\ln t & +\gamma) * \dot{E}(t ; A w(\alpha))-(\ln t+\gamma) I \\
& -A_{0} I_{t}^{\frac{d}{d \alpha} w(\alpha)} E(t ; A w(\alpha)) \\
& =-(\ln t+\gamma) I_{0} I_{t}^{\frac{d}{d \alpha} w(\alpha)} E(t ; A w(\alpha)) \\
& =-A^{-1}(\ln t+\gamma) * \dot{E}(t ; A w(\alpha)) .
\end{aligned}
$$

In the following, the solution of Eq. (29) is obtained in three special cases of the weight function. In the first case, a weight function is considered which consists of several weighted Dirac delta functions. This would turn Eq. (29) into a multi-term system of differential equations.

\section{Theorem 2}

The exact solution of Eq. (29) in the case of $w(\alpha)=$ $\sum_{i=1}^{n} r_{i} \delta\left(\alpha-\beta_{i}\right)$, where $0<\beta_{i}<1$ and $r_{i}>0$, is given by Eq. (63) in which:

$$
\begin{aligned}
& E(t ; A w(\alpha))=E_{\left(\beta_{1}, \beta_{2}, \ldots, \beta_{n}\right), 1} \\
& \quad\left(A r_{1} t^{\beta_{1}}, A r_{2} t^{\beta_{2}}, \ldots, A r_{n} t^{\beta_{n}}\right), \\
& t \geq 0 .
\end{aligned}
$$

\section{Proof}

The weight function in the above question has the following representation in the Laplace domain:

$$
L_{\alpha \rightarrow s}\{w(\alpha)\}=\sum_{i=1}^{n} r_{i} \exp \left(-s \beta_{i}\right) \text {. }
$$

By using the multinomial theorem [20], it is found that:

$$
\begin{gathered}
\left(L_{\alpha \rightarrow s}\{w(\alpha)\}\right)^{k}=\sum_{l_{1}+l_{2}+\cdots+l_{n}=k}\left(\begin{array}{c}
k \\
l_{1}, l_{2}, \cdots, l_{n}
\end{array}\right) \\
\prod_{i=1}^{n} r_{i}{ }^{l_{i}} \exp \left(-s l_{i} \beta_{i}\right)
\end{gathered}
$$




$$
\begin{aligned}
& =\sum_{l_{1}+l_{2}+\cdots+l_{n}=k}\left(\begin{array}{c}
k \\
l_{1}, l_{2}, \cdots, l_{n}
\end{array}\right) \\
& \exp \left(-s \sum_{i=1}^{n} l_{i} \beta_{i}\right) \prod_{i=1}^{n} r_{i}{ }^{l_{i}} .
\end{aligned}
$$

Thus, by considering the inverse Laplace transform, for iterated self-convolutions of the weight function, it is found that:

$$
\begin{aligned}
w^{* k}(\alpha)= & \sum_{l_{1}+l_{2}+\cdots+l_{n}=k}\left(\begin{array}{c}
k \\
l_{1}, l_{2}, \cdots, l_{n}
\end{array}\right) \prod_{i=1}^{n} r_{i}^{l_{i}} \delta \\
& \left(\alpha-\sum_{i=1}^{n} l_{i} \beta_{i}\right) .
\end{aligned}
$$

Thereby:

$$
\begin{gathered}
\int_{0}^{+\infty} w^{* k}(\alpha)\left(t^{\alpha} / \Gamma(\alpha+1)\right) d \alpha \\
=\sum_{l_{1}+l_{2}+\cdots+l_{n}=k}\left(\begin{array}{c}
k \\
l_{1}, l_{2}, \cdots, l_{n}
\end{array}\right) \prod_{i=1}^{n} r_{i}^{l_{i}} \\
\frac{t^{\sum_{i=1}^{n} l_{i} \beta_{i}}}{\Gamma\left(\sum_{i=1}^{n} l_{i} \beta_{i}+1\right)} .
\end{gathered}
$$

Finally, Based on Eq. (34), it is concluded that:

$$
\begin{aligned}
E(t ; A w(\alpha))= & \sum_{k=0}^{+\infty} \sum_{l_{1}+l_{2}+\cdots+l_{n}=k}\left(\begin{array}{c}
k \\
l_{1}, l_{2}, \cdots, l_{n}
\end{array}\right) \\
& A^{k} \prod_{i=1}^{n} r_{i}^{l_{i}} \frac{t^{\sum_{i=1}^{n} l_{i} \beta_{i}}}{\Gamma\left(\sum_{i=1}^{n} l_{i} \beta_{i}+1\right)}
\end{aligned}
$$

Since $l_{1}+l_{1}+\cdots+l_{n}=k$ holds in the inner summation, it is possible to write:

$$
\begin{aligned}
& E(t ; A w(\alpha))=\sum_{k=0}^{+\infty} \sum_{l_{1}+l_{2}+\cdots+l_{n}=k}\left(\begin{array}{c}
k \\
l_{1}, l_{2}, \cdots, l_{n}
\end{array}\right) \\
& \prod_{i=1}^{n}\left(A r_{i}\right)^{l_{i}} \frac{t^{\sum_{i=1}^{n} l_{i} \beta_{i}}}{\Gamma\left(\sum_{i=1}^{n} l_{i} \beta_{i}+1\right)} \\
& \quad=\sum_{k=0}^{+\infty} \sum_{l_{1}+l_{2}+\cdots+l_{n}=k}\left(\begin{array}{c}
k \\
l_{1}, l_{2}, \cdots, l_{n}
\end{array}\right) \\
& \prod_{i=1}^{n}\left(A r_{i} t^{\beta_{i}}\right)^{l_{i}} / \Gamma\left(\sum_{i=1}^{n} l_{i} \beta_{i}+1\right) \\
& =E_{\left(\beta_{1}, \beta_{2}, \ldots, \beta_{n}\right), 1}\left(A r_{1} t^{\beta_{1}}, A r_{2} t^{\beta_{2}}, \ldots, A r_{n} t^{\beta_{n}}\right) .
\end{aligned}
$$

\section{Theorem 3}

The solution of Eq. (29) in the case:

$$
w(\alpha)= \begin{cases}1, & \alpha \in(0,1) \\ 0, & \alpha \in(-\infty, 0] \cup[1,+\infty)\end{cases}
$$

is given by Eq. (63) in which:

$$
\begin{aligned}
& E(t ; A w(\alpha)) \\
& \quad=I+\exp (A) t W_{1,2}(-A t)-\ln t \int_{0}^{+\infty} W_{1, \beta+1} \\
& \quad(-A t \beta) \exp (A \beta) t^{\beta} d \beta, \quad t>0 .
\end{aligned}
$$

\section{Proof}

First of all, the Laplace transform of self-convolutions of the weight function is calculated as follows:

$$
\begin{aligned}
& W(s)=(1-\exp (-s)) / s, \\
& W^{k}(s)=(1-\exp (-s))^{k} / s^{k} .
\end{aligned}
$$

Using binomial theorem [20], we obtain:

$$
W^{k}(s)=\sum_{i=0}^{k}\left(\begin{array}{c}
k \\
i
\end{array}\right)(-1)^{i} \exp (-s i) / s^{k} .
$$

In order to find the solution Eq. (63) expressed in terms of Eq. (34), $v(\alpha+1)$ should be calculated by considering the inverse Laplace transform of Eq. (33) as follows:

$$
v(\alpha+1)=\delta(\alpha) I+\sum_{k=1}^{+\infty} A^{k} L_{s \rightarrow \alpha}^{-1}\left\{W^{k}(s)\right\} .
$$

According to Eqs. (86) and Eq. (87), it is deduced that:

$$
\begin{aligned}
v(\alpha+1)= & \delta(\alpha) I+\sum_{i=0}^{+\infty} A^{k} \sum_{i=0}^{k}\left(\begin{array}{l}
k \\
i
\end{array}\right) \\
& (-1)^{i}(\alpha-i)^{k-1} H(\alpha-i) / \Gamma(K) .
\end{aligned}
$$

By changing the order of series, we can write:

$$
\begin{aligned}
v(\alpha+1)= & \delta(\alpha) I+\sum_{i=0}^{+\infty} \sum_{k=i}^{+\infty} A^{k} \\
& \left(\begin{array}{c}
k \\
i
\end{array}\right)(-1)^{i} k(\alpha-i)^{k-1} H(\alpha-i) / k !
\end{aligned}
$$

Note that in the range of $\alpha \in(m, m+1]$, where $m \in \mathbb{Z}^{\geq 0}$, there holds:

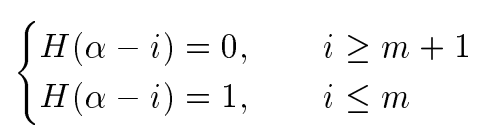


Thus, we only need to consider $i \leq m$ in the series (89) which results in:

$$
\begin{aligned}
v(\alpha+1)= & \delta(\alpha) I+\sum_{i=0}^{m} \sum_{k=i}^{+\infty} A^{k}\left(\begin{array}{c}
k \\
i
\end{array}\right) \\
& (-1)^{i} k(\alpha-i)^{k-1} / k !, \alpha \in(m, m+1],
\end{aligned}
$$

$m \in \mathbb{Z}^{\geq} 0$.

Changing the variables as $j=k-i$, we obtain:

$$
\begin{aligned}
v(\alpha+1)= & \delta(\alpha) I+\sum_{i=0}^{m} \sum_{j=0}^{+\infty} \\
& A^{i+j}((i+j) / i ! j !)(-1)^{i}(\alpha-i)^{i+j-1} \\
= & \delta(\alpha) I+\sum_{i=0}^{m}\left((-A)^{i}(\alpha-i)^{i-1} / i !\right) \\
& \sum_{j=0}^{+\infty} A^{j}(i+j)(\alpha-i)^{j} / j !, \\
& \alpha \in(m, m+1], \quad m \in \mathbb{Z}^{\geq 0} .
\end{aligned}
$$

The inner series in Eq. (92) equals:

$$
\begin{aligned}
& \sum_{j=0}^{+\infty} A^{j}(i+j)(\alpha-i)^{j} / j ! \\
&= i \sum_{j=0}^{+\infty} A^{j}(\alpha-i)^{j} / j ! \\
&+\sum_{j=1}^{+\infty} A^{j}(\alpha-i)^{j} /(j-1) !
\end{aligned}
$$

The first series in Eq. (93) is already expressed by the exponential function series. Changing the variable as $j^{\prime}=j-1$ in the second series makes it possible to express both series by:

$$
\begin{aligned}
& \sum_{j=0}^{+\infty} A^{j}(i+j)(\alpha-i)^{j} / j ! \\
& \quad=(i I+A(\alpha-i)) \exp (A(\alpha-i)) .
\end{aligned}
$$

Replacing Eq. (94) in Eq. (92) gives:

$$
\begin{gathered}
v(\alpha+1)=\delta(\alpha) I+\sum_{i=0}^{m} g(\alpha, i), \\
\alpha \in(m, m+1], \quad m \in \mathbb{Z}^{\geq 0},
\end{gathered}
$$

in which:

$$
\begin{aligned}
g(\alpha, i)= & (-A)^{i}(\alpha-i)^{i-1}(i I+A(\alpha-i)) \\
& \exp (A(\alpha-i)) / i !
\end{aligned}
$$

Therefore, according to Eqs. (34) and (95), after some manipulation, it is possible to write the solution as follows:

$$
\begin{aligned}
E(t ; A w(\alpha))= & I+\sum_{i=0}^{+\infty} \int_{i}^{+\infty} \\
& g(\alpha, i)\left(t^{\alpha} / \Gamma(\alpha+1)\right) d \alpha .
\end{aligned}
$$

Changing the variable as $\beta=\alpha-i$ in the integral in the right side of Eq. (97) results in:

$$
\begin{aligned}
& E(t ; A w(\alpha)) \\
& \quad=I+\int_{0}^{+\infty} \sum_{i=0}^{+\infty} g(\beta+i, i)\left(t^{\beta+i} / \Gamma(\beta+i+1)\right) d \beta .
\end{aligned}
$$

Replacing $g(\beta+i, i)$ with its value obtained by Eq. (96) yields:

$$
\begin{aligned}
E(t ; A w(\alpha))= & I+\int_{0}^{+\infty} \sum_{i=0}^{+\infty} \\
& (-A)^{i} \beta^{i-1}(i I+A \beta) \exp (A \beta)\left(t^{\beta+i} / \Gamma\right. \\
& (\beta+i+1)) / i ! d \beta .
\end{aligned}
$$

Splitting the series in Eq. (99) gives:

$$
\begin{aligned}
E(t ; A w(\alpha))= & I+\int_{0}^{+\infty} t^{\beta} \exp (A \beta) \\
& \left(\sum_{i=1}^{+\infty} \frac{(-A)^{i} t^{i} \beta^{i-1} i}{i ! \Gamma(\beta+i+1)}\right. \\
& \left.+\sum_{i=0}^{+\infty} \frac{(-A)^{i} t^{i} \beta^{i} A}{i ! \Gamma(\beta+i+1)}\right) d \beta .
\end{aligned}
$$

The second series in Eq. (100) is already a Wright function. Changing the variable as $i^{\prime}=i-1$ in the first series makes it possible to express both series in terms of the Wright function. By doing so, we obtain:

$$
\begin{aligned}
& E(t ; A w(\alpha))= \\
& I+\int_{0}^{+\infty} t^{\beta} \exp (A \beta)\left(-A t W_{1, \beta+2}(-A \beta t)\right. \\
& \left.\quad+A W_{1, \beta+1}(-A \beta t)\right) d \beta .
\end{aligned}
$$

Since there holds $W_{\alpha, \beta+\alpha}(z)=\frac{d}{d z} W_{\alpha, \beta}(z)$ for the Wright function, Eq. (101) can be written in the following form:

$$
\begin{aligned}
& E(t ; A w(\alpha))= \\
& I+\int_{0}^{+\infty} t^{\beta} \exp (A \beta) \frac{d}{d \beta}\left(W_{1, \beta+1}(-A \beta t)\right) d \beta \\
& +A \int_{0}^{+\infty} t^{\beta} \exp (A \beta) W_{1, \beta+1}(-A \beta t) d \beta,(102)
\end{aligned}
$$

which allows us to integrate the first term by parts. Doing so results in (83). 


\section{Corollary 1}

The solution of Eq. (29) in the case:

$$
\begin{aligned}
& w(\alpha)= \begin{cases}c a^{\alpha}, & \alpha \in(0,1) \\
0, & \alpha \in(-\infty, 0] \cup[1,+\infty)\end{cases} \\
& a \in \mathbb{R}^{+}, c \in \mathbb{R}
\end{aligned}
$$

is given by Eq. (63) in which:

$$
\begin{aligned}
& E(t ; A w(\alpha))=I+\exp (c A) a t W_{1,2}(-c a A t) \\
& -\ln a t \int_{0}^{+\infty} W_{1, \beta+1}(-c a A t \beta) \exp (c A \beta)(a t)^{\beta} d \beta,
\end{aligned}
$$

for $t>0$.

\section{Proof}

Let us denote the unitary weight function by:

$$
w_{1}(\alpha)= \begin{cases}1, & \alpha \in(0,1) \\ 0, & \alpha \in(-\infty, 0] \cup[1,+\infty)\end{cases}
$$

Then, the relation $w(\alpha)=c a^{\alpha} w_{1}(\alpha)$ holds between the two weight functions. Thus, by using the third part of Lemma 7 , it is deduced that:

$$
E(t ; A w(\alpha))=E\left(a t ; c A w_{1}(\alpha)\right) .
$$

Using Theorem 3 for the term $E\left(a t ; c A w_{1}(\alpha)\right)$ in Eq. (104) concludes the proof. $\square$

\section{Numerical examples}

In this section, at first, we will focus on the distributed order relaxation equation in the Riemann-Liouville sense as follows:

$$
\begin{gathered}
\dot{x}(t)=-\lambda \int_{0}^{1} w(\alpha)_{0}^{R L} D_{t}^{1-\alpha} x(t) d \alpha, \\
x(0)=1, \quad \lambda>0 .
\end{gathered}
$$

The solution of Eq. (105) in the cases of:

$$
\begin{aligned}
& w(\alpha)= \begin{cases}1, & \alpha \in(0,1) \\
0, & \alpha \in(-\infty, 0] \cup[1,+\infty)\end{cases} \\
& w(\alpha)= \begin{cases}\alpha, & \alpha \in(0,1) \\
0, & \alpha \in(-\infty, 0] \cup[1,+\infty)\end{cases} \\
& w(\alpha)= \begin{cases}\alpha^{2}, & \alpha \in(0,1) \\
0, & \alpha \in(-\infty, 0] \cup[1,+\infty)\end{cases}
\end{aligned}
$$

respectively, is shown in Figures 1, 2, and 3 for $\lambda \in$ $\{0.5,1,1.5,2\}$. (For numerically finding the solutions, at first, their representations in the Laplace domain are derived by using Lemma 6. Talbot's method [21] is utilized afterward as a numerical technique for inversion of the solutions back to the time domain.) As it can be seen in these figures, the solution decay is more intense for greater amounts of $\lambda$. In addition, the solutions associated with different weight functions are evaluated together in Figure 4 where $\lambda=1$.

As another example, the system of linear differential equations is considered:

$$
\begin{gathered}
\dot{x}(t)- \\
-{ }_{0}^{R L} D_{t}^{0.8} x(t)+{ }_{0}^{R L} D_{t}^{0.6} x(t)+{ }_{0}^{R L} D_{t}^{0.4} x(t) \\
\left.+{ }_{0}^{R L} D_{t}^{0.2} x(t)\right)=B g(t), \quad x(0)=x_{0},
\end{gathered}
$$

in which $A=\left[\begin{array}{cc}1 & -0.8 \\ 0.8 & 1\end{array}\right]$ and $B=\left[\begin{array}{l}0.25 \\ 0.25\end{array}\right]$. The

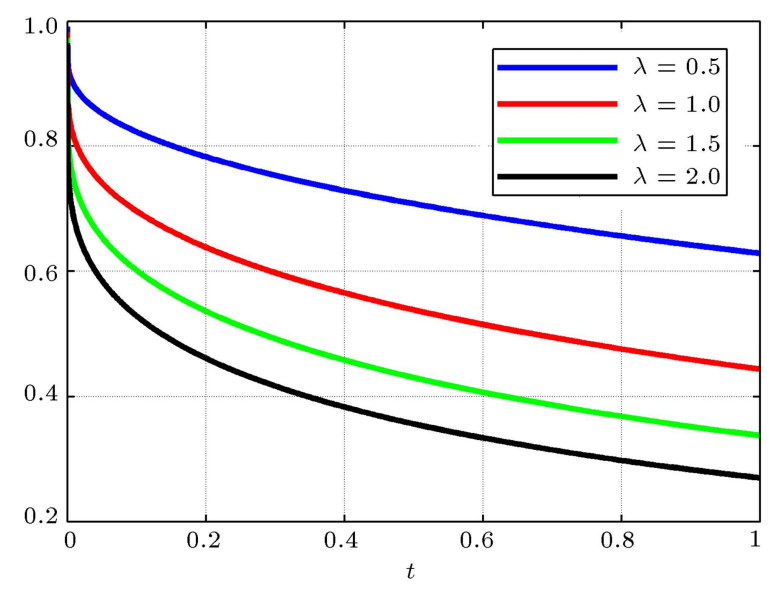

Figure 1. Solution of Eq. (105) with $w(\alpha)= \begin{cases}1, & \alpha \in(0,1) \\ 0, & \alpha \in(-\infty, 0] \cup[1,+\infty)\end{cases}$
for $\lambda \in\{0.5,1,1.5,2\}$.

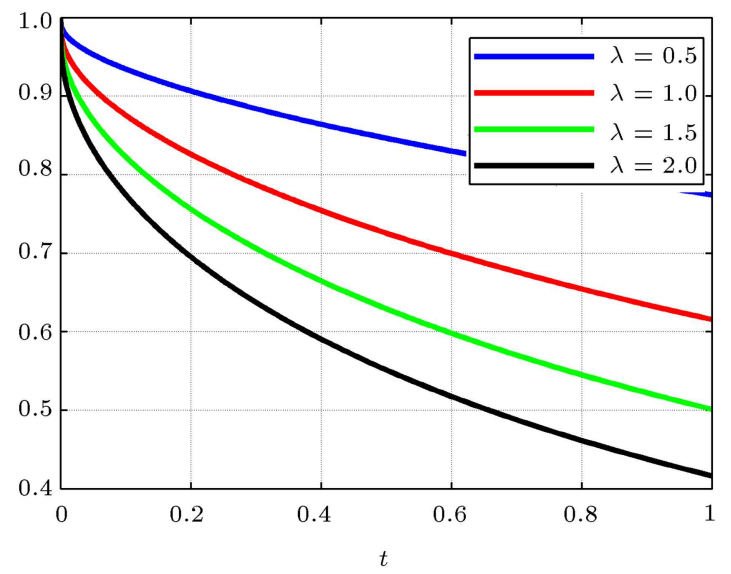

Figure 2. Solution of Eq. (105) with $w(\alpha)= \begin{cases}\alpha, & \alpha \in(0,1) \\ 0, & \alpha \in(-\infty, 0] \cup[1,+\infty)\end{cases}$
for $\lambda \in\{0.5,1,1.5,2\}$. 


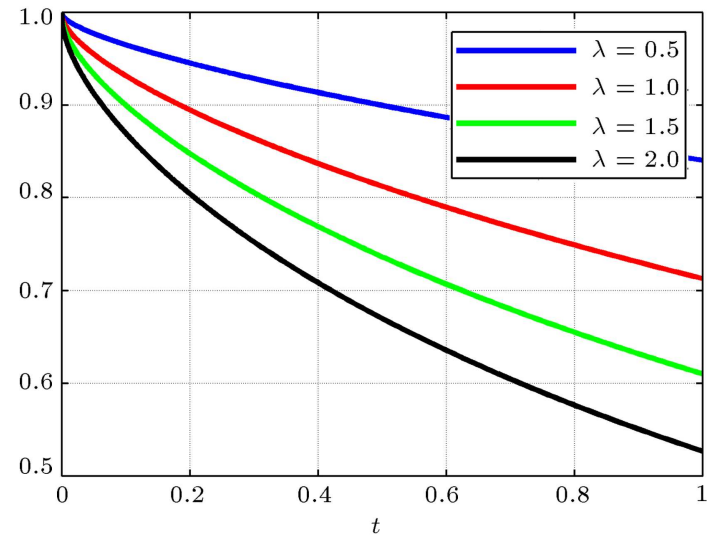

Figure 3. Solution of Eq. (105) with $w(\alpha)= \begin{cases}\alpha^{2}, & \alpha \in(0,1) \\ 0, & \alpha \in(-\infty, 0] \cup[1,+\infty)\end{cases}$ for $\lambda \in\{0.5,1,1.5,2\}$.

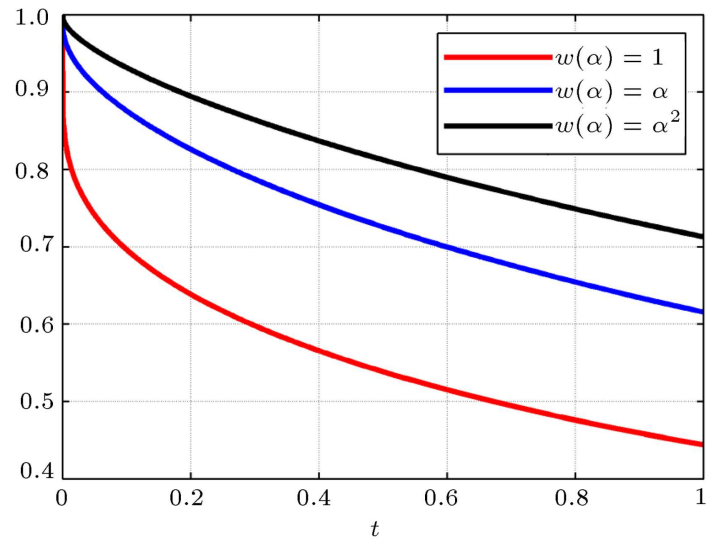

Figure 4. Solution of Eq. (105) for different weight functions where $\lambda=1$.

system of linear differential equations (106) can be rewritten in the form of the distributed order system of linear differential equations (29) by choosing the weight function $w(\alpha)=\delta(\alpha-0.2)+\delta(\alpha-0.4)+\delta(\alpha-0.6)+$ $\delta(\alpha-0.8)$. Therefore, its solution is given by Theorem 1. Assume that $x_{0}=(1 / \sqrt{2}, 1 / \sqrt{2})^{T}$ and $g(t)=H(t)$. The solution in this case is indeed provided by the sum of homogeneous and particular solutions, which are, respectively, shown in Figures 5 and 6 .

\section{Conclusion}

The system of linear differential equations of distributed order defined in the Riemann-Liouville sense was studied in this paper. The analytic solution of such a system was presented in terms of what can be interpreted as the distributed order generalization of matrix Mittag-Leffler functions. Some interesting properties of this function were revealed, and it was shown that this function turns into the multivariate Mittag-Leffler function when the weight function is

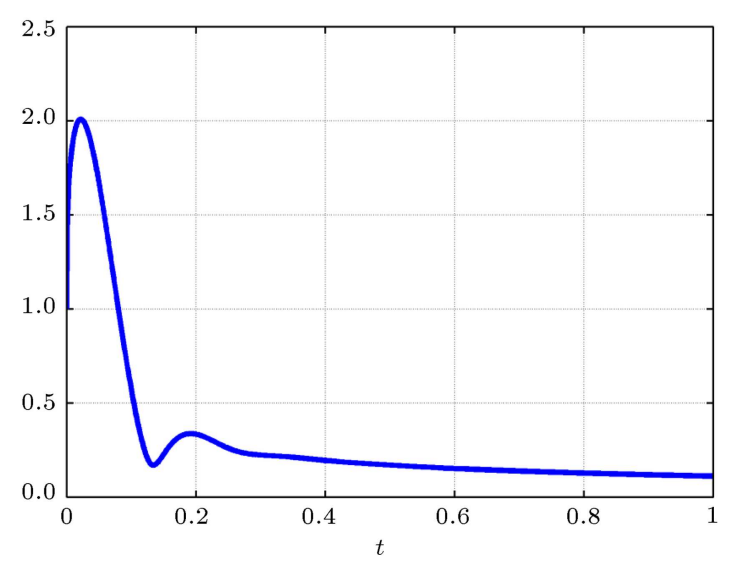

Figure 5. Solution of Eq. (106) where $x_{0}=(1 / \sqrt{2}, 1 / \sqrt{2})^{T}$ and $g(t)=0$.

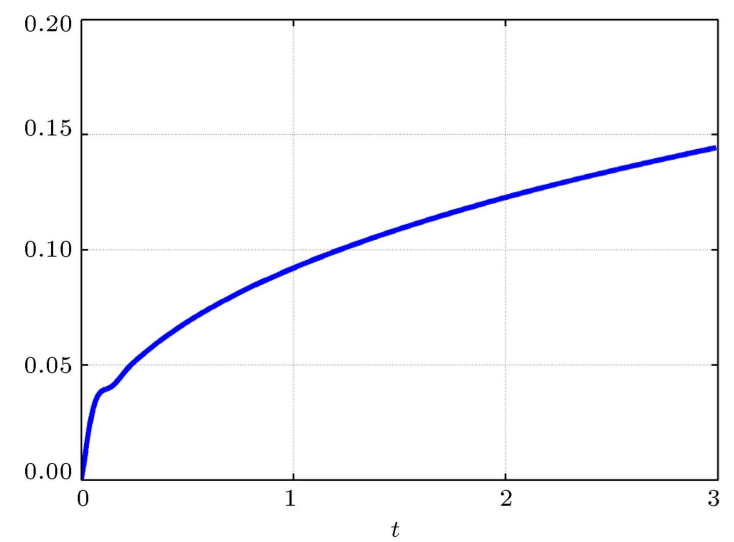

Figure 6. Solution of Eq. (106) where $x_{0}=(0,0)^{T}$ and $g(t)=H(t)$.

made up of several impulses and can be expressed in terms of the Wright function in the case of an exponential weight function. Since a special case of the problem considered in this paper is the distributed order relaxation equation, a numerical simulation was performed to evaluate the solutions to such equations. As another example, the solution of a system of differential equations with multiple fractional order operators was numerically obtained.

\section{References}

1. Rocca, M.C., Plastino, A.R., Plastino, A., Ferri, G.L., and de Paoli, A. "General solution of a fractional diffusion-advection equation for solar cosmic-ray transport", Physica A: Statistical Mechanics and its Applications, 447, pp. 402-410 (2016).

2. Bellouquid, A., Nieto, J., and Urrutia, L. "About the kinetic description of fractional diffusion equations modeling chemotaxis", Mathematical Models and Methods in Applied Sciences, 26(02), pp. 249-268 (2016).

3. Rosa, C.F.A.E. and Capelas de Oliveira, E. "Relaxation equations: fractional models", Journal of 
Physical Mathematics, 6(2) (2015).

DOI: $10.4172 / 2090-0902.1000146$

https://projecteuclid.org/euclid.jpm/1504144903

4. Saxena, R.K. and Pagnini, G. "Exact solutions of triple-order time-fractional differential equations for anomalous relaxation and diffusion I: The accelerating case", Physica A: Statistical Mechanics and Its Applications, 390(4), pp. 602-613 (2011).

5. Bobylev, A.V. and Cercignani, C. "The inverse Laplace transform of some analytic functions with an application to the eternal solutions of the Boltzmann equation", Applied Mathematics Letters, 15(7), pp. 807-813 (2002).

6. Mainardi, F., Mura, A., Gorenflo, R., and Stojanović, M. "The two forms of fractional relaxation of distributed order", Journal of Vibration and Control, 13(9-10), pp. 1249-1268 (2007).

7. Ansari, A. and Moradi, M. "Exact solutions to some models of distributed-order time fractional diffusion equations via the Fox H functions", Science Asia, 39, pp. 57-66 (2013).

8. Naber, M. "Distributed order fractional sub-diffusion", Fractals, 12(01), pp. 23-32 (2004).

9. Mainardi, F. and Pagnini, G. "The role of the FoxWright functions in fractional sub-diffusion of distributed order", Journal of Computational and Applied Mathematics, 207(2), pp. 245-257 (2007).

10. Stojanović, M. "Fractional relaxation equations of distributed order", Nonlinear Analysis: Real World Applications, 13(2), pp. 939-946 (2012).

11. Langlands, T.A.M. "Solution of a modified fractional diffusion equation", Physica A: Statistical Mechanics and Its Applications, 367, pp. 136-144 (2006).

12. Podlubny, I., Fractional differential equations: an introduction to fractional derivatives, fractional differential equations, to methods of their solution and some of their applications 198, Academic press, San Diego, USA (1998).

13. Li, Y., Sheng, H., and Chen, Y.Q. "On distributed order integrator/differentiator", Signal Processing, 91(5), pp. 1079-1084 (2011).

14. Erdelyi, A., Magnus, W., Oberhettinger, F., and Tricomi, F., Tables of Integral Transforms, 1 (1954).
15. Haubold, H.J., Mathai, A.M., and Saxena, R.K. "Mittag-Leffler functions and their applications", Journal of Applied Mathematics, 2011, Article ID 298628 (2011). DOI: $10.1155 / 2011 / 298628$

16. Gorenflo, R., Kilbas, A.A., Mainardi, F., and Sergei, V. Rogosin, Mittag-Leffler Functions, Related Topics and Applications, Springer, Berlin, Germany (2014).

17. Sandev, T., Chechkin, A.V., Korabel, N., Kantz, H., Sokolov, I.M., and Metzler, R. "Distributed-order diffusion equations and multifractality: Models and solutions", Physical Review E, 92(4), 042117 (2015).

18. Jiao, Z., Chen, Y.Q., and Podlubny, I., DistributedOrder Dynamic Systems: Stability, Simulation, Applications and Perspectives, Springer Briefs in Electrical and Computer Engineering (2012).

19. Chrouda, M.B., El Oued, M., and Ouerdiane, H. "Convolution calculus and applications to stochastic differential equations", Soochow Journal of Mathematics, 28(4), pp. 375-388 (2002).

20. Gossett, E., Discrete Mathematics with Proof, John Wiley \& Sons (2009).

21. Abate, J. and Whitt, W. "A unified framework for numerically inverting Laplace transforms", INFORMS Journal on Computing, 18(4), pp. 408-421 (2006).

\section{Biographies}

Hamed Taghavian is an MSc student majoring in Control Systems at the Department of Electrical Engineering at Sharif University of Technology. His research interests include fractional distributed order calculus and the relevant dynamic systems.

Mohammad Saleh Tavazoei received his BSc, MSc, and $\mathrm{PhD}$ in Electrical Engineering from the Sharif University of Technology, Tehran, Iran in 2003, 2005, and 2008, respectively. He is currently an Associate Professor at the Department of Electrical Engineering, Sharif University of Technology. His current research interests include dynamical behavior analysis of fractional order systems and applications of these systems in control system design. 